PROCEEDINGS OF THE

AMERICAN MATHEMATICAL SOCIETY

Volume 135, Number 7, July 2007, Pages 1987-1992

S 0002-9939(07)08723-0

Article electronically published on March 2, 2007

\title{
TRIPLE PRODUCT IDENTITY, QUINTUPLE PRODUCT IDENTITY AND RAMANUJAN'S DIFFERENTIAL EQUATIONS FOR THE CLASSICAL EISENSTEIN SERIES
}

\author{
HENG HUAT CHAN
}

(Communicated by Wen-Ching Winnie Li)

\begin{abstract}
In this article, we use the triple product identity and the quintuple product identity to derive Ramanujan's famous differential equations for the Eisenstein series.
\end{abstract}

\section{InTRODUCTION}

In his famous paper [10, S. Ramanujan gave elementary proofs to two trigonometric identities

$$
\begin{aligned}
& \left(\frac{1}{4} \cot \frac{u}{2}+\sum_{k=1}^{\infty} \frac{q^{k}}{1-q^{k}} \sin k u\right)^{2} \\
& =\left(\frac{1}{4} \cot \frac{u}{2}\right)^{2}+\sum_{k=1}^{\infty} \frac{q^{k}}{\left(1-q^{k}\right)^{2}} \cos k u+\frac{1}{2}\left(\sum_{k=1}^{\infty} \frac{k q^{k}}{1-q^{k}}(1-\cos k u)\right)
\end{aligned}
$$

and

$$
\begin{aligned}
& \left(\frac{1}{8} \cot ^{2} \frac{u}{2}+\frac{1}{12}+\sum_{k=1}^{\infty} \frac{k q^{k}}{1-q^{k}}(1-\cos k u)\right)^{2} \\
& =\left(\frac{1}{8} \cot ^{2} \frac{u}{2}+\frac{1}{12}\right)^{2}+\frac{1}{12} \sum_{k=1}^{\infty} \frac{k^{3} q^{k}}{1-q^{k}}(5+\cos k u),
\end{aligned}
$$

where $q=e^{2 \pi i \tau}, \operatorname{Im} \tau>0$. He then deduced from (1.1) and (1.2) many identities satisfied by the classical Eisenstein series

$$
E_{2 k}=1-\frac{4 k}{B_{2 k}} \sum_{n \geq 1} \frac{n^{2 k-1} q^{n}}{1-q^{n}},
$$

where $B_{2 k}$ is the Bernoulli number defined by

$$
\frac{x}{e^{x}-1}=\sum_{k \geq 0} B_{k} \frac{x^{k}}{k !}
$$

Received by the editors June 27, 2005 and, in revised form, December 27, 2005 and March 23, 2006.

2000 Mathematics Subject Classification. Primary 14K25.

(C)2007 American Mathematical Society Reverts to public domain 28 years from publication 
In particular, he showed that if $P=E_{2}(q), Q=E_{4}(q)$ and $R=E_{6}(q)$, then

$$
\begin{aligned}
& q \frac{d P}{d q}=\frac{P^{2}-Q}{12}, \\
& q \frac{d Q}{d q}=\frac{P Q-R}{3}
\end{aligned}
$$

and

$$
q \frac{d R}{d q}=\frac{P R-Q^{2}}{2} .
$$

Using (1.5), (1.6) and (1.7), Ramanujan derived the famous identity

$$
\eta^{24}(\tau):=q \prod_{k=1}^{\infty}\left(1-q^{k}\right)^{24}=\frac{1}{1728}\left(Q^{3}-R^{2}\right) .
$$

Identity (1.8) together with the fact that $Q$ and $R$ are respectively the normalization of the Eisenstein series

$$
G_{4}(\tau):=\sum_{\substack{(m, n) \in \mathbf{Z} \times \mathbf{z} \\(m, n) \neq(0,0)}} \frac{1}{(m \tau+n)^{4}}
$$

and

$$
G_{6}(\tau):=\sum_{\substack{(m, n) \in \mathbf{Z} \times \mathbf{z} \\(m, n) \neq(0,0)}} \frac{1}{(m \tau+n)^{6}}
$$

implies the transformation formula

$$
\eta(-1 / \tau)=(-i \tau)^{1 / 2} \eta(\tau)
$$

The purpose of this article is to derive new proofs of (1.5)-(1.7) using only the Jacobi triple product identity and the quintuple product identity.

For other proofs of (1.5)-11.7) with combinatorial flavor, see [12, 13] and 7].

For proofs of (1.5) - (1.7) using the theory of modular forms, see for example [8, p. 161, Theorem 5.3]. These proofs, however, require the knowledge of (1.9), as well as the dimension of the space of modular forms of weight 4,6 , and 8 on $\mathrm{SL}_{2}(\mathbf{Z})$.

\section{The Jacobi triple PROduCT IDENTITY}

The Jacobi theta function is defined by

$$
\vartheta_{1}(u \mid \tau)=2 \sum_{k=0}^{\infty}(-1)^{k} q^{(2 k+1)^{2} / 8} \sin (2 k+1) u .
$$

By Jacobi's triple product identity [1, p. 497, Theorem 10.4.1], we know that

$$
\vartheta_{1}(u \mid \tau)=2 q^{1 / 8} \sin u \prod_{k=1}^{\infty}\left(1-q^{k}\right)\left(1-q^{k} e^{2 i u}\right)\left(1-q^{k} e^{-2 i u}\right) .
$$

By logarithmically differentiating the above with respect to $u$, we find that

$$
\frac{\vartheta_{1}^{\prime}(u \mid \tau)}{\vartheta_{1}(u \mid \tau)}=\cot u+4 \sum_{k \geq 1} \frac{q^{k}}{1-q^{k}} \sin 2 k u .
$$


One can rewrite the above as (see [9, Lemma 2])

$$
\frac{\vartheta_{1}^{\prime}(u \mid \tau)}{\vartheta_{1}(u \mid \tau)}=\frac{1}{u}+\sum_{k \geq 1}(-1)^{k} \frac{2^{2 k} B_{2 k}}{(2 k) !} E_{2 k} u^{2 k-1},
$$

where $E_{2 k}$ and $B_{2 k}$ are given by (1.3) and (1.4), respectively.

Now, let

$$
S_{2 n+1}=2 \sum_{k=0}^{\infty}(-1)^{k}(2 k+1)^{2 n+1} q^{(2 k+1)^{2} / 8} .
$$

Then by expanding (2.1) in powers of $u$, we obtain

$$
\vartheta_{1}(u \mid \tau)=\sum_{n=0}^{\infty} \frac{(-1)^{n}}{(2 n+1) !} S_{2 n+1} u^{2 n+1} .
$$

Using (2.3) and (2.2), we find that

$$
\begin{aligned}
\sum_{n=0}^{\infty} \frac{(-1)^{n}}{(2 n) !} S_{2 n+1} u^{2 n}= & \left(\sum_{n=0}^{\infty} \frac{(-1)^{n}}{(2 n+1) !} S_{2 n+1} u^{2 n+1}\right) \\
& \times\left(\frac{1}{u}+\sum_{k \geq 1}(-1)^{k} \frac{2^{2 k} B_{2 k}}{(2 k) !} E_{2 k} u^{2 k-1}\right) .
\end{aligned}
$$

This implies that

$$
S_{2 n+1}=\frac{1}{2 n} \sum_{j=1}^{n} 2^{2 j}\left(\begin{array}{c}
2 n+1 \\
2 j
\end{array}\right) B_{2 j} E_{2 j} S_{2(n-j)+1} .
$$

The first four identities derived from this recurrence are:

$$
\begin{aligned}
& S_{3}=P S_{1}, \\
& S_{5}=\frac{5 P^{2}-2 Q}{3} S_{1}, \\
& S_{7}=\frac{35 P^{3}-42 P Q+16 R}{9} S_{1}
\end{aligned}
$$

and

$$
S_{9}=\left(\frac{35}{3} P^{4}-28 P^{2} Q+\frac{64}{3} P R+\frac{28}{5} Q^{2}-\frac{48}{5} E_{8}\right) S_{1},
$$

where we have replaced $E_{2}, E_{4}$ and $E_{6}$ by $P, Q$, and $R$, respectively.

Note that

$$
8 q \frac{d S_{n}}{d q}=S_{n+2} .
$$

Hence, by applying the operator $q \frac{d}{d q}$ to (2.4), we find that

$$
S_{5}=8 q \frac{d P}{d q} S_{1}+P S_{3}
$$

which implies that

$$
S_{5}=\left(8 q \frac{d P}{d q}+P^{2}\right) S_{1} .
$$

Comparing (2.8) with (2.5), we deduce (1.5). 
Similarly, by using (1.5), (2.5), (2.6) and (2.7), we deduce (1.6) and the identity

$$
-112 Q^{2}-320 P R+640 q \frac{d R}{d q}+432 E_{8}=0 .
$$

To prove (1.7), we need another relation similar to (2.9), and this will be given in the next section.

\section{The QuintuPle PRODUCT IDENTITY}

The quintuple product identity states that

$$
q^{1 / 24} \prod_{k=1}^{\infty}\left(1-q^{k}\right) \frac{\vartheta_{1}(2 u \mid \tau)}{\vartheta_{1}(u \mid \tau)}=2 \sum_{n=-\infty}^{\infty}(-1)^{n} q^{(6 n+1)^{2} / 24} \cos (6 n+1) u .
$$

One of the simplest proofs of an equivalent form of (3.1) is due to L. Carlitz and M.V. Subbarao 5. Their proof involves only the Jacobi triple product identity. For more details of the history and the different forms of (3.1), see [2, p. 83] or [6].

By expanding the right-hand side of (3.1) in powers of $u$, we deduce that

$$
q^{1 / 24} \prod_{k=1}^{\infty}\left(1-q^{k}\right) \frac{\vartheta_{1}(2 u \mid \tau)}{\vartheta_{1}(u \mid \tau)}=\sum_{k=0}^{\infty}(-1)^{k} \frac{T_{2 k}}{(2 k) !} u^{2 k}
$$

where

$$
T_{2 k}=2 \sum_{n=-\infty}^{\infty}(-1)^{n}(6 n+1)^{2 k} q^{(6 n+1)^{2} / 24} .
$$

By logarithmically differentiating both sides of (3.2), we find that

$$
\left(2 \frac{\vartheta_{1}^{\prime}}{\vartheta_{1}}(2 u \mid \tau)-\frac{\vartheta_{1}^{\prime}}{\vartheta_{1}}(u \mid \tau)\right)\left(\sum_{k=0}^{\infty}(-1)^{k} \frac{T_{2 k}}{(2 k) !} u^{2 k}\right)=\sum_{k=0}^{\infty}(-1)^{k+1} \frac{T_{2 k+2}}{(2 k+1) !} u^{2 k+1} .
$$

Using (2.2) and comparing the coefficients of powers of $u$ in (3.3), we find that for $n \geq 0$,

$$
T_{2 n+2}=\frac{1}{2 n+2} \sum_{j=0}^{n}\left(\begin{array}{l}
2 n+2 \\
2 j+2
\end{array}\right) 2^{2 j+2}\left(2^{2 j+2}-1\right) B_{2 j+2} E_{2 j+2} T_{2(n-j)} .
$$

We construct from (3.4) the following identities for $T_{2 k}, 1 \leq k \leq 4$ :

$$
\begin{aligned}
& T_{2}=P T_{0}, \\
& T_{4}=\left(3 P^{2}-2 Q\right) T_{0}, \\
& T_{6}=\left(15 P^{3}-30 P Q+16 R\right) T_{0}
\end{aligned}
$$

and

$$
T_{8}=\left(105 P^{4}-420 P^{2} Q+448 P R+140 Q^{2}-272 E_{8}\right) T_{0} .
$$

In the construction of the above identities, we have followed Z.G. Liu 911

Using (1.5), (1.6), (3.5), (3.6) and the relation

$$
24 q \frac{d T_{n}}{d q}=T_{n+2}
$$

\footnotetext{
${ }^{1}$ There is a misprint in the formula for $T_{8}$. The coefficient of $R P$ should be replaced by 336 .
} 
we find that

$$
-192 P R-80 Q^{2}+384 q \frac{d R}{d q}+272 E_{8}=0 .
$$

Solving the simultaneous equations (2.9) and (3.7), we deduce (1.7) and the identity

$$
E_{8}=Q^{2} \text {. }
$$

\section{Conclusion}

The functions $S_{2 k+1}$ and $T_{2 k}$ were studied by Ramanujan on page 369 of his Lost Notebook [11. Ramanujan showed that $S_{2 k+1} / S_{1}$ and $T_{2 k} / T_{0}$ can be expressed in terms of $P, Q$ and $R$. Further discussions of Ramanujan's proofs can be found in [14. pp. 31-32]. A discussion of $S_{2 k+1}$ can also be found in [3], while that of $T_{2 k}$ can be found in [4] and [9]. All the proofs discussed above use (1.5)-(1.7). We have shown here that in fact the first few identities satisfied by $S_{2 k+1} / S_{1}(k=1,2,3,4)$ and $T_{2 k} / T_{0}(k=1,2,3,4)$ are enough for us to deduce Ramanujan's results for these functions.

\section{ACKNOWLEDGMENTS}

The author sincerely thanks S. Cooper and the Institute for Information and Mathematical Sciences, Massey University (Albany Campus) for their generous support during his stay there. He is very grateful to S. Cooper for his encouragement and enthusiasm in this work.

\section{REFERENCES}

[1] G.E. Andrews, R. Askey and R. Roy, Special functions, Cambridge University Press, Cambridge, 1999. MR 1688958(2000g:33001)

[2] B.C. Berndt, Ramanujan's Notebooks, Part III, Springer-Verlag, New York, 1991. MR.1117903 (92j:01069)

[3] B. C. Berndt, S. H. Chan, Z.-G. Liu and H. Yesilyurt, A new identity for $(q ; q)_{\infty}^{10}$ with an application to Ramanujan's partition congruence modulo 11, Quart. J. Math., 55 (2004), 13-30. MR 2043004 (2004k:11166)

[4] B. C. Berndt and A. J. Yee, A page on Eisenstein series in Ramanujan's lost notebook, Glasgow Math. J., 45 (2003), 123-129. MR1972702 (2004a:11032)

[5] L. Carlitz and M.V. Subbarao, A simple proof of the quintuple product identity, Proc. Amer. Math. Soc. 32 (1972), 42-44. MR0289316 (44:6507)

[6] S. Cooper, The quintuple product identity, Int. J. Number Theory 2 (2006), 115-161. MR2217798

[7] J.G. Huard, Z.M. Ou, B.K. Spearman and K.S. Williams, Elementary evaluation of certain convolution sums involving divisor functions, in Number Theory for the Millennium, vol. 2, M.A. Bennett, B.C. Berndt, N. Boston, H.G. Diamond, A.J. Hildebrand and W. Philipp, eds., A K Peters, Natick, MA, 2002, pp. 229-274. MR1956253 (2003j:11008)

[8] S. Lang, Introduction to modular forms, Springer-Verlag, New York, 1995. MR:1363488 (96g:11037)

[9] Z.G. Liu, A three term theta function identity and its applications, Adv. Math. 195 (2005), 1-23. MR2145792 (2006c:11050)

[10] S. Ramanujan, On certain arithmetical functions, Trans. Cambridge Philos. Soc. 22 (1916), 159-184.

[11] S. Ramanujan, The lost notebook and other unpublished papers, Narosa, New Delhi, 1988. MR0947735 (89j:01078)

[12] R.A. Rankin, Elementary proofs of relations between Eisenstein series, Proc. Roy. Soc. Edinburgh 76A (1976) 107-117. MR 0441870(56:261)

[13] N.-D. Skoruppa, A quick combinatorial proof of Eisenstein series identities, J. Number Theory 43 (1993), 68-73. MR1200809 (94f:11029) 
[14] K. Venkatachaliengar, Development of elliptic functions according to Ramanujan, Technical Report, 2. Madurai Kamaraj University, Department of Mathematics, Madurai, 1988.

Department of Mathematics, National University of Singapore, 2 Science Drive 2, SINGAPORE 117543

E-mail address: matchh@nus.edu.sg 